\title{
Evaluación de la recarga producida mediante manejo de una masa de Pinus halepensis con técnicas de silvicultura hidrológica
}

\author{
Garcia-Prats, A.,"*, Del Campo-García, A. ${ }^{1}$, Molina, A.J. ${ }^{2}$ \\ ${ }^{1}$ Grupo de Investigación en Ciencia y Tecnología Forestal-REFOREST-. Departamento de Ingeniería Hidráulica \\ y Medio Ambiente. Universitat Politècnica de València. Camino de Vera s/n. 46022. Valencia.
}

${ }^{1}$ IRTA. Torre Marimón. 08140 Caldes de Montbui. Barcelona.

*e-mail: agprats@upvnet.upv.es

\section{Resumen}

El balance hídrico, y especialmente la recarga por percolación profunda de un suelo, puede ser modificado a nivel de parcela mediante el manejo de la masa. Extendido a nivel de cuenca debemos esperar que dicha modificación afecte al conjunto del ciclo hidrológico. Sin embargo, el primer paso es la cuantificación del efecto que produce sobre el balance de agua el manejo silvícola de la masa con fines hidrológicos a nivel de parcela. Para ello se diseñó un experimento en una parcela de Pinus halepensis en la que se realizaron aclareos de distinta intensidad. El ciclo del agua se monitorizó midiendo la temperatura, humedad relativa, pluviometría dentro y fuera de la masa, el contenido de agua del suelo y la transpiración. Para cuantificar el volumen de agua que atraviesa la zona de raíces se calibró y validó el modelo HYDRUS-1D, el cual resuelve las ecuaciones de flujo en medios porosos saturados y no saturados. Los resultados demuestran que la recarga conseguida mediante el manejo de la masa puede ser modificada, consiguiendo valores de recarga netamente superiores en las parcelas tratadas. Sin embargo reducciones por debajo del 50\% la fracción de cabida cubierta (FCC) no producen mejora en la recarga. Se comprueba que el régimen de precipitaciones tiene un efecto importante en el valor de la recarga. Dado que se trata de parcelas llanas en las que no se evidenció escorrentía, el estudio debe ser ampliado a laderas en donde este componente del ciclo presente una mayor importancia.

Palabras clave: Recarga subterránea, flujo no saturado, HYDRUS-1D, silvicultura hidrológica. 


\section{Introducción}

El agua subterránea es un recurso especialmente valioso es zonas áridas y semiáridas. Una gestión sostenible de dichos recursos es un reto que debe alcanzarse lo antes posible, ya que la sobreexplotacion actual de algunas zonas pone en riesgo los ecosistemas dependientes del agua subterránea. La dinámica de la vegetación en estas regiones climáticas es totalmente dependiente del régimen de humedad del suelo, siendo el agua el factor limitante para su desarrollo. Trabajos recientes sobre silvicultura adaptativa en masas mediterráneas de Pinus halepensis han puesto de manifiesto el papel fundamental del agua (Molina y Del Campo, 2012; Ungar et al., 2013; Del Campo et al., 2014) en esos ecosistemas. La evapotranspiración es el componente cuantitativamente más importante en el balance de agua de una cuenca, por lo que cualquier cambio en la estructura de la vegetación que afecte a dicho componente, obligatoriamente debe afectar al balance de agua y en último lugar a la recarga subterránea por percolación profunda (Chen et al., 2014). van Dijk et al. (2007) plantean una revisión de trabajos acerca de los efectos que tiene la forestación de tierras agrícolas a nivel de cuenca en los que parece existir un consenso que en climas estacionales el efecto generalizado es una disminución de caudales medios y bajos de los ríos (water yield) por el incremento de la evapotranspiración y el descenso de la recarga.

Por otro lado, la recarga subterránea puede producirse de distintas formas. Por una lado está la recarga difusa, relacionada con la percolación del agua precipitada a lo largo de toda la superficie permeable de la cuenca, mientras que por otro tiene lugar la recarga localizada, asociada a estructuras concretas como cauces perdedores, lagos, embalses, recargas artificiales etc. (Barron et al., 2012). Realizar mediciones directas de la recarga es especialmente complicado, por lo que es habitual recurrir a modelos matemáticos o combinaciones de ellos. Estos modelos podemos clasificarlos en función de la escala, de forma que hay modelos como SWAT o SWIM que trabajan a nivel de cuenca, modelos como MODFLOW que trabajan a nivel de acuífero, o modelos que resuelven el flujo poroso en medio no saturado o modelos de humedad del suelo como HYDRUS, SWAP o WAVES cuya escala de aplicación sería la parcela. Los modelos que trabajan a nivel de parcela y de acuífero suelen ser modelos de base física que resuelven las ecuaciones de flujo de agua en el medio poroso saturado y no saturado, por lo que es posible calibrarlos empleando variables fáciles de medir para obtener la recarga de forma inversa. Wattenbach et al. (2007) evalúan el efecto hidrológico de la forestación de tierras agrícolas y el cambio de especies en Alemania a nivel de cuenca utilizando SWIM. Dawes et al. (2012) obtienen la recarga de varios acuíferos empleando MODFLOW para los casos en los que dispone de datos para su calibración, y WAVES para aquellos casos en los que solo tiene datos a nivel de parcela. Chen et al. (2014) emplea también el modelo WAVES para evaluar la recarga subterránea en una zona semiárida de Australia. Hashemi et al. (2013) emplea MODFLOW para comparar un sistema de recarga artificial con otro natural. Guan et al. (2010) evalúan la percolación profunda en una ladera de Pinus ponderosa a nivel de parcela, para lo cual emplea HYDRUS; por citar algunas referencias recientes. 
Si nos centramos en los modelos que evalúan la recarga a nivel de parcela, es habitual emplear una serie de datos de campo equivalentes a los resultados que el modelo genera, y que se emplean para calibrar y validar el modelo. Así por ejemplo Chen et al., (2014) emplean series de valores medidos de índice de área foliar (LAI), evapotranspiración (ET) y contenido de agua del suelo (SWC) para calibrar WAVES.

El objetivo del presente trabajo es la cuantificación de todos las componentes del ciclo hidrológico con especial énfasis en la recarga, a nivel de parcela, en función del manejo silvícola que recibe la masa. El modelo que se empleó para obtener de forma inversa la recarga fue HYDRUS-1D. Para la calibración y validación del modelo se midieron series continuas de contenido de agua en el suelo (SWC), transpiración (T), y medidas puntuales de LAI.

\section{Material y métodos}

\subsection{Descripción del área de estudio}

El área de estudio es la misma que la descrita en Del Campo et al. (2014). Se ubicó en el Monte de La Hunde y Palomeras (3905’30” N, 1²'30” W), localizado al sureste de la provincia de Valencia (España) a $950 \mathrm{~m}$ de altura sobre el nivel del mar. El clima es Mediterráneo con una precipitación media anual de $466 \mathrm{~mm}$ y una temperatura media anual de $13.7^{\circ} \mathrm{C}$ (1960-2007). La evapotranspiración potencial es de $749 \mathrm{~mm} /$ año (Thornthwaite) y la evapotranspiración de referencia (ETo) de 1200 $\mathrm{mm}$ (Hargreaves). Los suelos tienen una elevada concentración en carbonato cálcico (26-38\%), un $\mathrm{pH}$ que oscila en el rango 7.7-8.2, y una profundidad que varía entre 50 y $60 \mathrm{~cm}$, presentando una textura franco-limo-arenosa. La pendiente de zona es muy baja, inferior al $5 \%$ en todas las parcelas.

\subsection{Diseño experimental}

Se realizó un diseño experimental de bloques completos al azar. Se plantearon 4 bloques de 0,36 ha separados menos de 200 m entre sí, los cuales contenían todos los tratamientos. La masa estudiada procede de una repoblación realizada en los años 40. Cada bloque se dividió en 4 parcelas de 30×30 m. Una parcela en cada bloque no recibió ningún aclareo de pies, presentando una fracción de cabida cubierta casi del 100\% y en un claro estado de estancamiento. Las otras 3 parcelas recibieron un tratamiento de aclareo de pies basado en la eliminación de aquellos pies menos desarrollados hasta obtener otras tres fracciones de cabida cubierta, que finalmente resultó como se resume en la Tab. 1. Las parcelas fueron inventariadas en marzo de 2009.

En cada una de las parcelas se midieron humedad relativa (HR), temperatura (Temp), precipitación (P), trascolación (THR), contenido de agua del suelo (SWC) y transpiración (T). Por otro lado, se realizó una medida puntual de índice de área foliar (LAI). 
Tabla 1. Estructura de la masa. $\mathrm{DBH}$ es diámetro medio a la altura del pecho, BA es el área basal. LAI es índice de área foliar. Adaptado de Molina y Del Campo (2012) y Del Campo et al. (2014).

\begin{tabular}{|c|c|c|c|c|c|c|}
\hline Tratamiento & $\begin{array}{c}\text { Cobertura } \\
(\%)\end{array}$ & $\begin{array}{c}\text { Densidad } \\
(\text { arboles ha-1 })\end{array}$ & $\begin{array}{c}\text { DBH } \\
(\mathrm{cm})\end{array}$ & $\begin{array}{c}\text { Altura Media } \\
(\mathrm{m})\end{array}$ & $\begin{array}{c}\text { BA } \\
\left(\mathrm{m}^{2} \mathrm{ha}^{-1}\right)\end{array}$ & $\begin{array}{c}\text { LAI } \\
\left(\mathrm{m}^{2} \mathrm{~m}^{-2}\right)\end{array}$ \\
\hline T100 (control) & 84 & 1489 & $17.8 \pm 5.1$ & 11.5 & 40.1 & 2.6 \\
\hline T60 (Intensidad Baja) & 68 & 744 & $21.2 \pm 4.1$ & 12.2 & 27.2 & 1.9 \\
\hline T30 (Intensidad Media) & 50 & 478 & $17.5 \pm 3.6$ & 11.3 & 18.2 & 1.5 \\
\hline T10 (Intensidad Alta) & 22 & 178 & $20.4 \pm 1.6$ & 12.2 & 9.4 & 0.5 \\
\hline
\end{tabular}

Temp y HR se midieron con un registrador de temperatura y humedad Hobo (HOBO U12 Temp RH Logger), instalado a $2 \mathrm{~m}$ de altura con registro de datos cada 15 minutos.

P se midió en una zona abierta separada $150 \mathrm{~m}$ de las parcelas experimentales con un pluviografo de cazoletas (Davis, USA). THR en cada parcela experimental se midió mediante unos canalones de $400 \mathrm{~cm}$ x $13,17 \mathrm{~cm}$ de ancho y elevados $50 \mathrm{~cm}$ del suelo instalados en la diagonal de las parcelas. Estos canalones recolectaron el agua en bidones de plástico. El agua se recolectó después de cada evento de precipitación, de forma que se aseguró que en ningún caso se superaba la capacidad del recipiente, lo que hubiera supuesto pérdida de datos. Para más detalles ver Molina y del Campo (2012).

SWC fue medido de forma continua para el periodo completo de referencia $(01 / 04 / 09-31 / 05 / 11)$ en todos los tratamientos cada 20 minutos mediante sensores FDR (EC-TM, Decagon Devices Inc., Pullman, WA). En cada tratamiento, entre 6 y 9 sensores fueron instalados a $30 \mathrm{~cm}$ de profundidad, teniendo en cuenta si existía o no influencia de algún árbol. Se realizaron calibraciones en campo determinando la humedad gravimétrica en cuatro puntos experimentales: saturación, capacidad de campo y punto de marchitez permanente con el objeto de obtener el rango completo del SWC en el lugar de estudio. La capacidad de campo se calculó a partir de los promedios de las lecturas de SWC en tres fechas en las que la lluvia fue superior a 30 mm en los 2 días previos. Para más detalles ver Molina y del Campo (2012).

El flujo de savia se midió utilizando el método HRM (Burgess et al., 2001; Hernandez-Santana et al., 2011; Williams et al., 2004) en cuatro árboles por tratamiento, durante el mismo periodo antes mencionado para SWC, y programado para registrar datos cada media hora. Los sensores de flujo de savia (HRM, ICT International, Australia) se instalaron en cada árbol seleccionado en la cara norte del tronco a 1.3 $\mathrm{m}$ de altura. El método se basa en un calentador que emite un pulso de calor y el incremento de temperatura que produce es medido en dos agujas separadas de forma equidistante $5 \mathrm{~mm}$ aguas arriba y aguas abajo del emisor de calor, las cuales poseen dos termopares localizados a dos profundidades: $27.5 \mathrm{~mm}$ (interior) y $12.5 \mathrm{~mm}$ (exterior). Cada par de medidas de temperatura es utilizada para estimar la velocidad del pulso de calor en las dos profundidades y es transformado en una medida de velocidad del flujo de savia. (Burgess et al., 2001). El área conductora de savia se obtuvo 
mediante la extracción de cores de madera con barrena en cada árbol con sensor de flujo de savia, y medidos en laboratorio con técnicas dendrocronológicas, empleando para ello un equipo LINTAB 6.0 y el software TSAP-win.

El LAI se estimó empleando un sensor LAI-2000 de LICOR. Los detalles de las mediciones con este equipo pueden consultarse en Molina y Del Campo (2012). Dado que se requieren medidas de LAI mensuales y solo se realizó una medida puntual de LAI, se recurren a curvas de evolución de LAI medidas a lo largo del año para la misma especie en la literatura. Así en Sprintsin et al. (2011), el LAI es medido mensualmente en varias parcelas de Pinus halepensis a lo largo de 2 años, proponiendo una curva de LAI anual para el semiárido mediterráneo. Tomando estos valores como relativos, se extrapola nuestro valor puntual al resto del año.

\subsection{Modelización del balance de agua con HYDRUS-1D}

HYDRUS-1D (Simunek et al., 2013) es un paquete de software que puede ser empleado para simular el movimiento del agua, solutos y calor de forma unidimensional en medio poroso parcial o totalmente saturado. Básicamente el programa resuelve numéricamente la ecuación unidimensional de Richards para el flujo de agua en medio poroso, comúnmente expresada como:

$$
\frac{\partial \theta(h)}{\partial t}=\frac{\partial}{\partial x}\left[K(h) \frac{\partial h}{\partial x}+K(h)\right]-S(h)
$$

Donde $h$ es el potencial hidráulico $(\mathrm{cm}) ; \theta$ es el contenido volumétrico de agua del suelo -SWC- $\left(\mathrm{cm}^{3} \mathrm{~cm}^{-3}\right) ; K$ es la conductividad hidráulica del suelo $\left(\mathrm{cm} \mathrm{d}^{-1}\right) ; t$ es el tiempo (d); $x(\mathrm{~cm})$ es la coordenada vertical la cual se expresa positiva hacia arriba; y $S$ es el término sumidero $\left(\mathrm{cm}^{3} \mathrm{~cm}^{-3} \mathrm{~d}^{-1}\right)$, el cual se utiliza para tener en cuenta la extracción de agua por parte de las raíces de las plantas.

Las funciones hidráulicas del suelo $\theta(h)$ y $K(h)$ expresan respectivamente las relaciones no lineales entre el contenido de agua del suelo y el potencial hidráulico por un lado y la conductividad hidráulica y el potencial por otro. HYDRUS permite el empleo de diferentes modelos de curvas. En nuestro caso se empleó el modelo de van Genuchten (1980)-Mualem (1976). Las curvas de retención de agua y de conductividad para el suelo vienen expresadas de la siguiente forma:

$$
\begin{gathered}
\theta(h)=\theta_{r}+\left(\theta_{s}-\theta_{r}\right)\left[1+(a h)^{\mathrm{n}}\right]^{-\mathrm{m}} \\
K(h)=K_{s} S_{e}\left[1-\left(1-S_{e}^{1 / \mathrm{m}}\right)^{\mathrm{m}}\right]^{2}
\end{gathered}
$$

donde $\theta_{r}$ y $\theta_{s}$ los contenidos de agua residual y en saturación $\left(\mathrm{cm}^{3} \mathrm{~cm}^{-3}\right)$, respectivamente; $\alpha\left(\mathrm{cm}^{-1}\right), n(-)$, y $m(-)$ son parámetros de forma de las curvas sabiendo que $m=1-1 / n ; K_{s}$ es la conductividad hidráulica saturada $\left(\mathrm{cm} \mathrm{d}^{-1}\right) ; l(-)$ es el factor de conectividad entre poros; $S_{e}(-)$ es la saturación efectiva expresada como: 


$$
S_{e}=\frac{\theta-\theta_{r}}{\theta_{s}-\theta_{r}}
$$

Finalmente, la transpiración real es obtenida según el modelo S-shaped de van Genuchten (1987), en el cual la transpiración potencial es reducida por un factor reductor que depende del potencial hidráulico existente en el suelo en cada momento, es decir el factor reductor por estrés hídrico:

$$
\begin{gathered}
T=\alpha(h) \cdot S_{p} \\
\alpha(h)=\frac{1}{1+\left(\frac{h}{h_{50}}\right)^{p}}
\end{gathered}
$$

Donde, $\mathrm{T}\left(\mathrm{cm} \mathrm{d}^{-1}\right)$ es la transpiración real, $S p\left(\mathrm{~cm} \mathrm{~d}^{-1}\right)$ es la tasa de transpiración potencial, $h_{50}(\mathrm{~cm})$ es el potencial hidráulico al cual la extracción de agua por parte de las raíces se reduce un 50\% y $p$ (-) es un parámetro experimental.

Para más detalle acerca del modelo ver Simunek et al. (2013).

\subsection{Datos de entrada}

\section{Datos geométricos}

HYDRUS construye un modelo geométrico de elementos finitos dividiendo el espesor total del perfil del suelo en 100 capas, siendo el espesor de cada una de ellas, la centésima parte del espesor total. En nuestro caso el espesor total de suelo encontrado es de $60 \mathrm{~cm}$, compuesto por un horizonte $\mathrm{O}$ de mantillo de $10 \mathrm{~cm}$ de espesor y un horizonte A de $50 \mathrm{~cm}$ de suelo ocupado por raíces. Bajo este horizonte se encontró directamente roca caliza con un elevado grado de karstificación que permite el drenaje libre. El nivel freático no fue encontrado, por lo que se modela con una condición de contorno en la base del perfil de drenaje libre.

\section{Características hidráulicas del suelo}

Los parámetros hidráulicos efectivos del suelo se obtuvieron por modelación inversa en HYDRUS. Para tener unos parámetros iniciales aproximados y poder fijar los intervalos de variación razonable, se empleó el modelo ROSETTA que establece las propiedades hidráulicas de un suelo en base a parámetros físicos del mismo. En este caso se empleó la textura.

\section{Condiciones de contorno}

Se establecieron como condiciones de contorno el flujo en superficie: evaporación, transpiración y escorrentía superficial y el flujo en la base del perfil: drenaje libre. Para la obtención de la extracción de agua por las raíces se empleó el modelo S-Shaped de van Genuchten, siendo los parámetros definitivos del mismo obtenidos por modelación inversa.

Como condiciones de contorno temporales se introdujeron los valores de TRH 
para cada día del periodo simulado.

\section{Parámetros meteorológicos}

Se introdujeron como datos de entrada de tipo meteorológico las temperaturas máxima y mínima, la altura de la vegetación (Tab. 1), el albedo (0.14), y la curva mensual de LAI extrapolada del valor puntual medido, según Sprintsin et al. (2011). El propio modelo obtiene los valores potenciales de evaporación y transpiración aplicando la fórmula de Hargreaves para utilizarlos como condición de contorno del flujo superficial. Por último la profundidad radicular definida fue de $60 \mathrm{~cm}$, igual a la profundidad total de suelo existente.

\section{Condiciones iniciales}

Se estableció como condición inicial el SWC medido el primer día de simulación.

\subsection{Calibración y validación del modelo}

La calibración se llevó a cabo con las mediciones de transpiración (T) y de contenido de agua en el suelo a la profundidad de $30 \mathrm{~cm}$ donde se instalaron los sensores (SWC) realizadas en el periodo 01/04/2009 hasta 31/03/2010. Los parámetros seleccionados para ajustar los resultados del modelo a los datos medidos en campo fueron las características hidráulicas del suelo según el modelo de van Genuchten $(\theta r$, $\theta s, \alpha, n)$ y los parámetros del modelo de extracción de agua del suelo S-Shaped de van Genuchten ( $n_{50} \mathrm{y} p$ ). El ajuste de los parámetros del modelo se desarrolló utilizando PEST (A model-independent parameter estimation program) (Doherty, 2009). PEST emplea el algoritmo de calibración no lineal denominado Gauss-Marquardt-Levenberg. La función objetivo minimiza el error cuadrático medio entre los valores simulados y observados de T y SWC dentro del periodo señalado, todos ellos con un mismo peso. PEST realiza un análisis de sensibilidad de las variables, resultando todas ellas con cierto grado de sensibilidad respecto del resultado final.

La validación del modelo se llevó a cabo aplicando de nuevo el modelo con los parámetros calibrados a un nuevo periodo de medición de las mismas variables diferente al usado en la calibración, concretamente de 01/04/2010 a 31/05/2011.

\subsection{Evaluación del funcionamiento del modelo}

Los resultados del modelo obtenidos en la fase de calibración y validación fueron evaluados por comparación con los resultados realmente medidos. Los estadísticos empleados a tal efecto fueron Eficiencia del modelo de Nash y Sutcliffe $E$ (Nash and Sutcliffe, 1970), Índice de ajuste de Willmott $d$ (Willmott, 1981) e Índice de ajuste de Willmott modificado $d_{1}$ (Willmott, 1984) y el error cuadrático medio RMSE.

\section{Eficiencia del modelo $E$ de Nash and Sutcliffe}

Eficiencia del modelo $E$ de Nash and Sutcliffe (1970) se define como 1 menos la suma de las diferencias absolutas al cuadrado entre los valores observados y pre- 
dichos, normalizado por la varianza de los valores observados durante el periodo investigado. Se calcula con la expresión:

$$
E=1-\frac{\sum_{i=1}^{h}\left(O_{i}-P_{i}\right)^{2}}{\sum_{i=1}^{n}(O-\bar{O})^{2}}
$$

Donde $O_{i}$, valor observado el día i; $P_{i}$, el predicho por el modelo el día i; y finalmente $O$ es el valor medio de los valores observados.

El rango entre el cual $E$ puede oscilar va desde 1 a $-\infty .1$ indica un ajuste perfecto, mientras que cero indica que el valor medio de las observaciones es igual predictor que el propio modelo. Valores negativos indican que el valor medio de las observaciones es mejor predictor que el propio modelo.

Una de las desventajas del índice $E$ de Nash-Sutcliffe radica en que emplea los cuadrados de las diferencias entre los valores predichos y observados, lo que conduce a sobrevalorar valores extremos y a despreciar valores pequeños (Legates and McCabe, 1999).

Índice de ajuste de Willmot

El índice de ajuste de Willmot $d$ (Willmot, 1981) representa el ratio entre el error cuadrático medio y el error potencial (Willmot, 1984) y se define como:

$$
d=1-\frac{\sum_{i=1}^{n}\left(O_{i}-O_{i}\right)^{2}}{\sum_{i=1}^{n}\left(\left|P_{i}-\bar{O}\right|+\left|O_{i}-O\right|\right)^{2}}
$$

El índice $d$ toma valores que oscilan entre 0 (sin correlación alguna) hasta 1 (ajuste perfecto).

El error potencial en el denominador representa el mayor valor que las diferencias cuadráticas de cada par de valores puede tomar. Al presentar las diferencias al cuadrado en el numerador, presenta el mismo inconveniente que $E$ al sobrevalorar los valores extremos y despreciar los valores bajos.

\section{Índice de ajuste de Willmot modificado}

El índice de ajuste modificado $d_{1}$ fue desarrollado por Willmot (1984) para aumentar la sensibilidad con valores bajos y evitar sobrevalorar en exceso los valores extremos, se expresa de la siguiente forma:

$$
d_{1}=1-\frac{\sum_{i=1}^{n}\left|O_{i}-O_{i}\right|}{\sum_{i=1}^{n}\left(\left|P_{i}-\bar{O}\right|+\left|O_{i}-\bar{O}\right|\right)}
$$

El rango en el que puede oscilar el índice $d_{1}$ es el mismo que $d$.

\section{Error cuadrático medio (RMSE)}

RMSE es una medida del error medio, ponderado de acuerdo al cuadrado del error. Oscila entre 0 a $+\infty$, siendo 0 el ajuste perfecto sin error. Se calcula como: 


$$
R M S E=\sqrt{\frac{1}{n} \sum_{i=1}^{n}\left(P_{i}-O_{i}\right)^{2}}
$$

Donde $n=$ número de observaciones.

\section{Resultados}

\subsection{Parámetros efectivos del modelo}

En la Tab. 2 quedan resumidos los parámetros efectivos obtenidos en el proceso de calibración y que permiten ajustar los resultados modelados de SWC y T a los observados en campo.

Tabla 2. Parámetros hidráulicos efectivos para el modelo de van Genuchten-Mualem y para el modelo de extracción de agua por las raíces S-Shaped de van Genuchten obtenidos en el periodo de calibración. $\theta_{r}$ y $\theta_{s}$ : contenidos de agua residual y en saturación $\left(\mathrm{cm}^{3} \mathrm{~cm}^{-3}\right)$, respectivamente; $\alpha\left(\mathrm{cm}^{-1}\right), n(-)$, y $m(-)$ : parámetros de forma de las curva de humedad donde $m=1-1 / n ; K$ : conductividad hidráulica saturada (cm d-1); 1 (-) es el factor de conectividad entre poros; $S_{e}(-)$ : saturación efectiva; $h_{50}(\mathrm{~cm})$ : potencial hidráulico al cual la extracción de agua por parte de las raíces se reduce un $50 \%$ y $p(-)$ : parámetro experimental de ajuste de la curva de extracción de agua.

\begin{tabular}{|c|c|c|c|c|c|c|c|c|}
\hline $\begin{array}{c}\text { Tratamiento } \\
\mathbf{c m}^{\mathbf{3}} \mathbf{c m}^{-3}\end{array}$ & $\begin{array}{c}\theta \mathbf{r} \\
\mathbf{c m}^{3} \mathbf{c m}^{-3}\end{array}$ & $\begin{array}{c}\theta \mathbf{s} \\
\mathbf{c m}^{-1}\end{array}$ & $\begin{array}{c}\alpha \\
(-)\end{array}$ & $\begin{array}{c}\mathbf{n} \\
\mathbf{c m ~ \mathbf { d } ^ { - 1 }}\end{array}$ & $\begin{array}{c}\mathbf{K}_{\mathbf{s}} \\
(-)\end{array}$ & $\begin{array}{c}\mathbf{I} \\
(\mathbf{c m})\end{array}$ & $\begin{array}{c}\mathbf{h}_{50} \\
(-)\end{array}$ & $\mathbf{p}$ \\
\hline T100 (control) & 0.08226 & 0.43516 & 0.075291 & 1.420 & 38.22 & 0.5 & -44.40 & 0.7123 \\
\hline T60 (Intensidad Baja) & 0.08226 & 0.43516 & 0.015000 & 2.066 & 9.13 & 0.5 & -107.32 & 0.9689 \\
\hline T30 (Intensidad Media) & 0.08226 & 0.43516 & 0.073055 & 1.376 & 20.74 & 0.5 & -200.01 & 0.1000 \\
\hline T10 (Intensidad Alta) & 0.08226 & 0.43516 & 0.033830 & 1.836 & 13.96 & 0.5 & -33.32 & 1.0798 \\
\hline
\end{tabular}

\subsection{Comparación entre resultados medidos y modelados}

La Tab. 3 recoge los resultados obtenidos en los estadísticos de evaluación del funcionamiento del modelo, tanto en el proceso de calibración como en la posterior validación. La eficiencia del modelo ajustado según Nash y Sutcliffe oscila entre 0.35 y 0.83 en calibración y entre 0.42 y 0.67 en validación. El índice de Willmott obtenido oscila entre 0.60 y 0.94 en calibración y entre 0.83 y 0.91 en validación. El índice de Willmott modificado varía entre 0.4 y 0.79 para la fase de calibración y entre 0.61 y 0.70 en la de validación. Por último el error cuadrático medio oscila entre 0.1 y 0.21 al comparar valores de $\mathrm{T}$, oscilando entre 0.03 a 0.04 al comparar valores medidos y modelados de SWC. En general se acepta que los modelos funcionan correctamente cuando $\mathrm{E}>0.35 \mathrm{y} \mathrm{d}-\mathrm{d}_{1}>0.5$.

La comparación entre datos medidos y modelados, para el periodo completo de referencia podemos observarlo gráficamente en la Fig. 1, tanto para SWC (1-a) como 
para T (1-b). La comparación gráfica, más allá del valor resumido del estadístico, sirve para detectar periodos en los que el ajuste no ha sido satisfactorio. En nuestro caso se observa en general un funcionamiento razonable salvo en periodos puntuales en los que SWC se ha acercado al punto de marchitez permanente que el modelo predijo valores ligeramente superiores de SWC.

Tabla 3. Evaluación de funcionamiento del modelo. Nash y Sutcliffe E, Índice de ajuste de Willmott $d$, Índice de ajuste de Willmott modificado $d_{1}$ y error cuadrático medio RMSE. En fase de calibración $C$. En fase de validación $V$.

\begin{tabular}{|c|c|c|c|c|c|c|c|c|}
\hline \multirow[t]{2}{*}{ Tratamiento } & \multicolumn{2}{|c|}{$\mathbf{E}$} & \multicolumn{2}{|c|}{ d } & \multicolumn{2}{|c|}{ d1 } & \multicolumn{2}{|c|}{ RMSE } \\
\hline & $\mathrm{C}$ & $\mathbf{V}$ & $\mathbf{C}$ & $\mathbf{V}$ & $\mathrm{C}$ & $\mathbf{V}$ & $\mathbf{C}$ & $\mathbf{V}$ \\
\hline $\mathrm{T} 100(\mathrm{~T})$ & 0.40 & 0.62 & 0.79 & 0.86 & 0.57 & 0.66 & 0.21 & 0.21 \\
\hline T60 (T) & 0.53 & 0.51 & 0.84 & 0.83 & 0.62 & 0.61 & 0.14 & 0.15 \\
\hline $\mathrm{T} 30(\mathrm{~T})$ & 0.35 & 0.42 & 0.60 & 0.84 & 0.40 & 0.65 & 0.14 & 0.10 \\
\hline $\mathrm{T} 10(\mathrm{~T})$ & 0.60 & 0.42 & 0.85 & 0.83 & 0.66 & 0.62 & 0.15 & 0.15 \\
\hline \multirow[t]{2}{*}{ Tratamiento } & \multicolumn{2}{|c|}{$\mathbf{E}$} & \multicolumn{2}{|c|}{ d } & \multicolumn{2}{|c|}{ d1 } & \multicolumn{2}{|c|}{ RMSE } \\
\hline & $\mathrm{C}$ & $\mathbf{V}$ & $\mathbf{C}$ & $\mathbf{V}$ & $\mathrm{C}$ & $\mathbf{V}$ & $\mathbf{C}$ & $\mathbf{V}$ \\
\hline T100 (SWC) & 0.76 & 0.52 & 0.91 & 0.86 & 0.75 & 0.62 & 0.04 & 0.03 \\
\hline T60 (SWC) & 0.83 & 0.54 & 0.94 & 0.87 & 0.79 & 0.70 & 0.03 & 0.03 \\
\hline T30 (SWC) & 0.68 & 0.51 & 0.89 & 0.82 & 0.70 & 0.60 & 0.04 & 0.04 \\
\hline T10 (SWC) & 0.82 & 0.67 & 0.94 & 0.91 & 0.77 & 0.69 & 0.04 & 0.03 \\
\hline
\end{tabular}

\subsection{Evaluación de la recarga}

Con el modelo calibrado y validado atendemos a los resultados obtenidos en la recarga según el tratamiento de aclareo que recibe la masa. La evolución a lo largo de los dos años de mediciones se muestran gráficamente en la Fig. 2. Los resultados numéricos se comparan en la Tab. 4. Si bien el periodo completo de mediciones comprende desde 01/04/2009 al 31/05/2011, y tal y como se indicó anteriormente, se ha empleado el primer año (01/04/2009 - 31/03/2010) para la calibración y el resto del periodo para la validación $(01 / 04 / 2010$ - 31/05/2011), a la hora de comparar datos se ha prescindido de los últimos dos meses, de forma que el segundo periodo tenga exactamente los mismos días que el primero. Por tanto el segundo periodo estudiado comprende de 01/04/2010 a 01/04/2011.

El efecto de aclareo produjo un incremento de la translocación que osciló de los $409 \mathrm{~mm}$ del control T100 sin tratar hasta los $624 \mathrm{~mm}$ para el máximo aclareo de T10 el primer año, y de $362 \mathrm{~mm}$ (T100) a $558 \mathrm{~mm}$ (T10) el segundo año. Sin embargo esto el primer año se tradujo en una recarga que osciló entre los 124 mm de T100 y los 227 mm de T10, mientras que para el segundo año la recarga solo varió desde los $26 \mathrm{~mm}$ de T100 a los 90 mm de T10, manteniéndose las tasas de transpiración muy simila- 


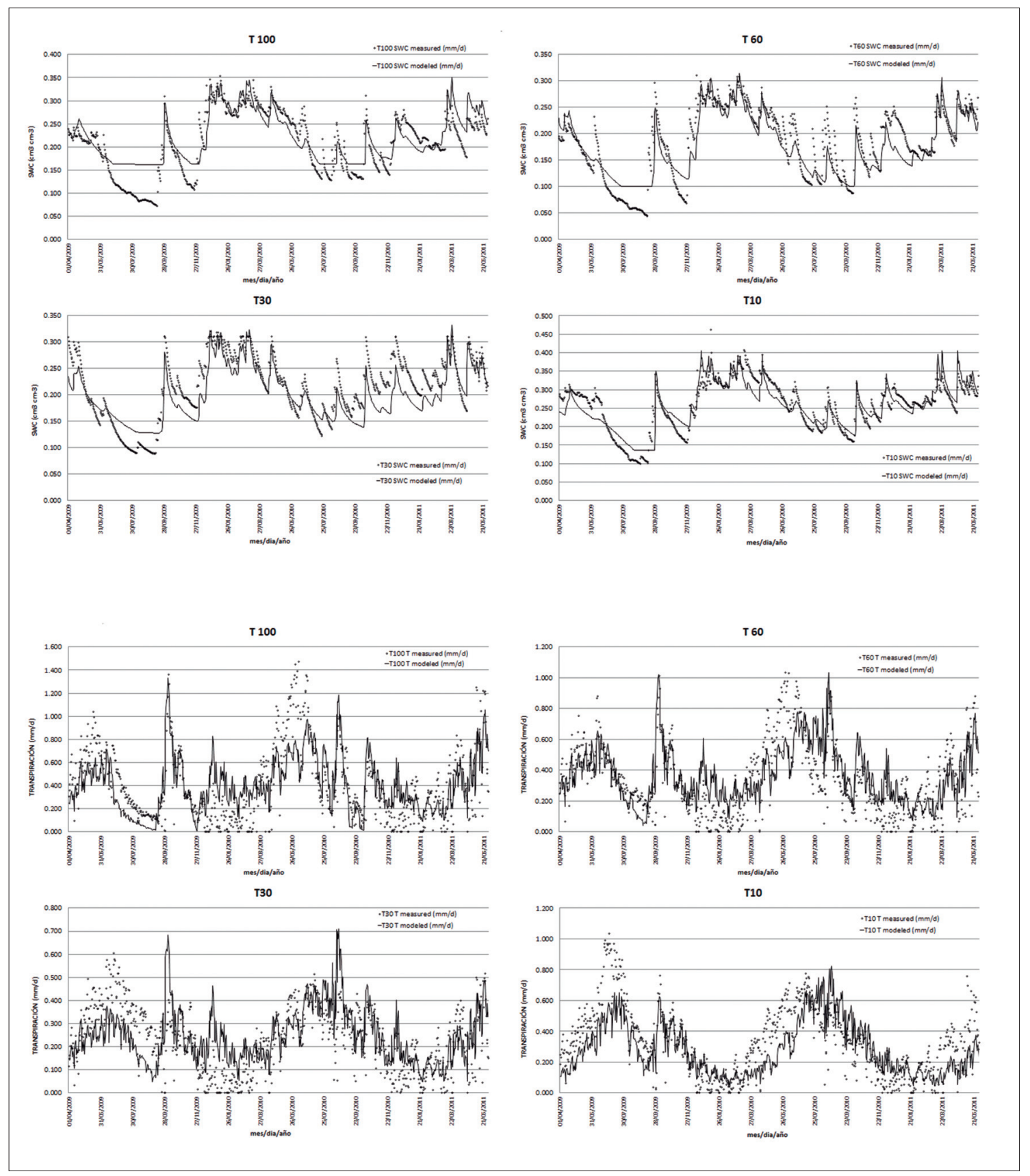

Figura 1. Resultados de HYDRUS-1D para T y SWC. Periodo de calibración abril/2009 - marzo/2010. Periodo de validación abril/2010-mayo/2011.

res en las parcelas tratadas. Sin embargo en el control, la transpiración del primer año fue superior a la del segundo año.

HYDRUS-1D evalúa la escorrentía superficial en base a la precipitación, de forma que solo se produce ésta cuando la precipitación supera la capacidad de infiltración del suelo evaluada en cada momento con la ecuación de flujo. En todos los casos la escorrentía fue nula, aspecto este que se pudo comprobar en campo, no ha- 


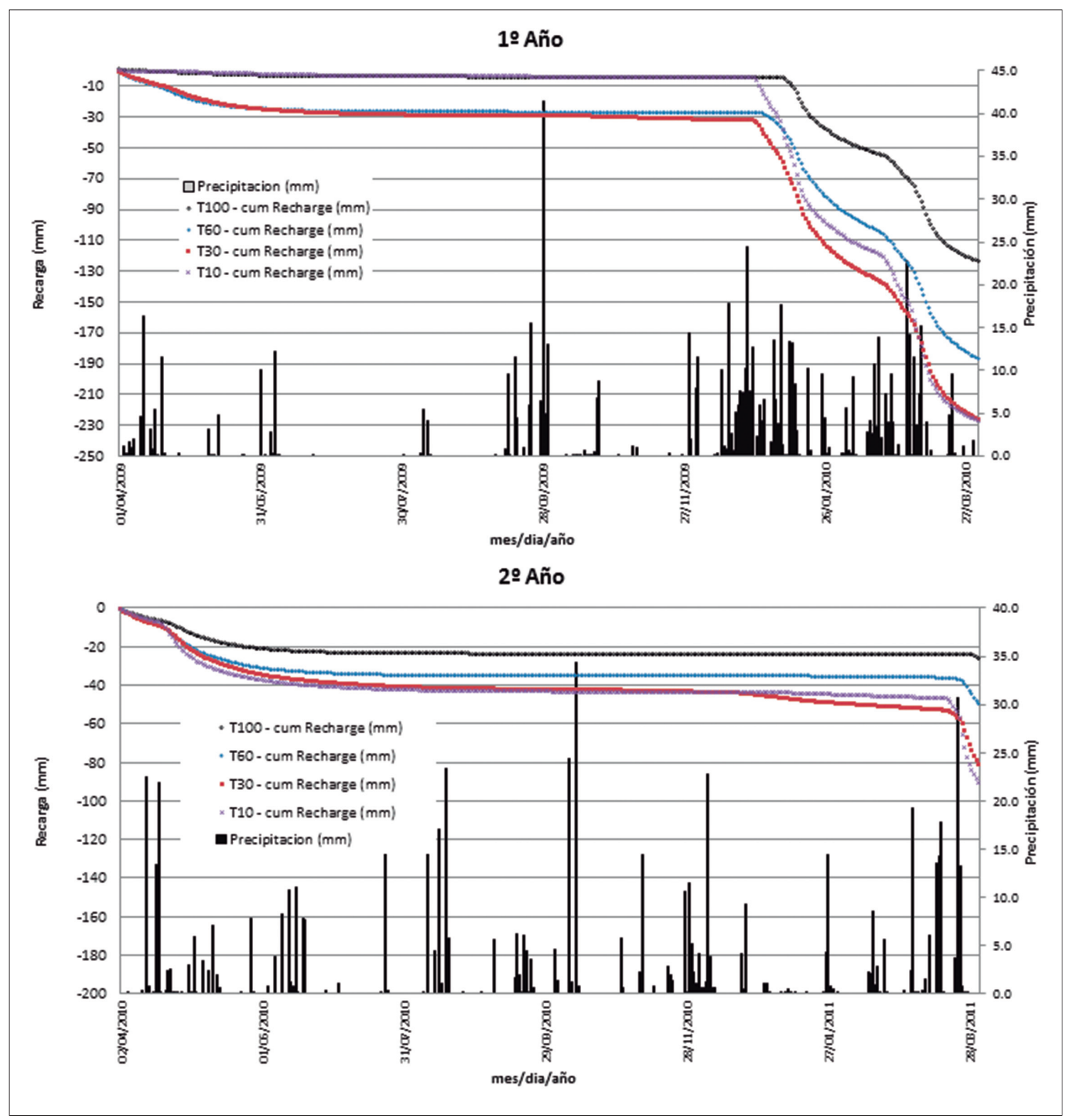

Figura 2. Recarga producida según tratamiento y año.

Tabla 4. Comparación del balance hídrico por periodos y tratamiento.

\begin{tabular}{|c|c|c|c|c|c|c|c|c|}
\hline Tratamiento & \multicolumn{2}{|c|}{$\begin{array}{c}\text { P-Precipitación } \\
(\mathbf{m m})\end{array}$} & \multicolumn{2}{|c|}{$\begin{array}{c}\text { THR-Translocación } \\
(\mathbf{m m})\end{array}$} & \multicolumn{2}{|c|}{$\begin{array}{c}\text { Recarga } \\
(\mathbf{m m})\end{array}$} & \multicolumn{2}{c|}{$\begin{array}{c}\text { T-Transpiración } \\
(\mathbf{m m})\end{array}$} \\
\hline & Año 1 & Año 2 & Año 1 & Año 2 & Año 1 & Año 2 & Año 1 & Año 2 \\
\hline T10 & 690 & 618 & 624 & 558 & 227 & 90 & 81 & 83 \\
\hline T30 & 690 & 618 & 511 & 454 & 226 & 81 & 110 & 114 \\
\hline T60 & 690 & 618 & 491 & 436 & 187 & 50 & 126 & 129 \\
\hline T100 & 690 & 618 & 409 & 362 & 124 & 26 & 125 & 153 \\
\hline
\end{tabular}


biendo evidencia alguna de que se generase escorrentía superficial a lo largo del periodo de medición.

\section{Discusión}

En general el modelo se puede decir que funcionó adecuadamente en base a los estadísticos empleados. Este funcionamiento, como suele ocurrir en estos casos, fue sustancialmente mejor para SWC que para T. Esto no solo es atribuible al funcionamiento del modelo, sino también a la incertidumbre asociada a las medidas de campo. Hay que tener en cuenta que las medidas de transpiración se realizan de forma puntual en un árbol y el resultado es una velocidad de flujo de savia. Esta velocidad se debe transformar en caudal multiplicando por el área conductora, lo que lleva asociado un determinado error que seguramente es significativo, y finalmente este caudal se promedia con el de todos los árboles monitorizados, que deben ser representativos de la masa. Esto hace que estas medidas lleven asociado un importante grado de incertidumbre. No obstante, aun así es muy importante incluirlas en la calibración del modelo, pues la alternativa es estimar la transpiración de forma totalmente empírica (Jhorar et al., 2004). Teniendo en cuenta que el modelo explica de forma más que satisfactoria el SWC, entendemos que el balance hídrico obtenido puede ser indicativo del efecto hidrológico que tiene un determinado manejo de la masa.

En el primer periodo la precipitación fue superior al segundo. Esto se tradujo, en función del tratamiento recibido por la masa, en que THR fue mayor en las masas con una mayor intensidad de aclareo. Por otro lado se comprueba gráficamente que el régimen de precipitaciones también fue diferente, de forma que las precipitaciones el primer año están más agrupadas en el otoño y la primavera, mientras que en el segundo están temporalmente repartidas a lo largo del año. Sin embargo, las recargas del primero año son muy superiores al segundo en valor absoluto, manteniéndose las transpiraciones en valores muy parecidos. Es decir, el descenso o incremento de precipitación fue directamente a recarga, no modificándose la transpiración de forma sustancial. Hay que decir que fueron dos años especialmente lluviosos, en los que seguramente la transpiración estuvo muy cerca de valores potenciales, y que en años en los que la precipitación se acerque más al promedio de la zona (466 mm), la transpiración tendrá un peso específico mayor, produciéndose un descenso importante de la recarga.

Podemos deducir pues que el efecto del tratamiento de la masa es claro en la recarga, más evidente en términos relativos en años menos lluviosos, y que el régimen de las precipitaciones es especialmente importante. Lluvias de menor cantidad y más repartidas a lo largo del año van a engrosar el capítulo de la intercepción primero y de la transpiración después, mientras que lluvias abundantes concentradas en otoño y primavera intensifican el fenómeno de la recarga.

También es importante indicar que el efecto del incremento de recarga producido por el manejo de la masa con aclareos pierde su efecto a partir de un cierto grado 
de intensidad. En nuestro caso produjeron similares recargas las intensidades T10 y T30 correspondientes a coberturas de 22 y $50 \%$ respectivamente.

En ningún caso se produjo escorrentía superficial, seguramente por la poca pendiente de las parcelas. En un futuro el experimento deberá replicarse en parcelas en donde dicha componente aparezca de forma obligatoria para evaluar su peso en el balance.

\section{Conclusiones}

En el presente trabajo se planteó un diseño experimental para evaluar el efecto sobre el balance hídrico que tiene un tratamiento de aclareo en una masa artificial procedente de repoblación y en un claro estado de estancamiento. Dado que la recarga es un componente del balance especialmente complejo de medir, se evaluó por modelación inversa con el modelo HYDRUS-1D. Los estadísticos de funcionamiento del modelo fueron especialmente buenos en SWC, y aceptables en T. Las conclusiones a las que se llega de forma esquemática son las siguientes:

- El manejo de la masa produce un incremento en la recarga, el cual es tanto más importante en términos relativos, cuanto menor es la precipitación.

- Por debajo de coberturas del 50\% la recarga no se ve incrementada, y seguramente otros procesos no evaluados como la protección y conservación del suelo se ven comprometidos.

- El régimen de precipitaciones es muy importante. Lluvias abundantes concentradas en periodo de reposo incrementa la recarga, frente a periodos con lluvias de poca entidad mucho más repartidas en el tiempo.

- Durante el periodo de estudio las lluvias fueron especialmente abundantes, por lo que la traspiración se acercó a valores potenciales durante muchos periodos. El ensayo debería replicarse con periodos de precipitación más parecidos a la media de la zona.

- Las parcelas elegidas son especialmente llanas, lo que sin duda contribuyó a que no se generase escorrentía superficial. El ensayo debería replicarse en un futuro con parcelas que aseguren que dicho componente no sea nulo, para evaluar el efecto en la recarga.

\section{Agradecimientos}

Este studio forma parte del Proyecto de investigación: "CGL2011-28776-C0202 , Hydrological characterization of forest structures at plot scale for an adaptive management, HYDROSIL", financiado por el Ministerio de Ciencia e Innovación y fondos FEDER. 


\section{Bibliografía}

Barron, O. V., Crosbie, R. S., Dawes, W. R., Charles, S. P., Pickett, T., Donn, M. J., 2012. Climatic Controls on Diffuse Groundwater Recharge Across Australia. Hydrol. Earth Syst. Sci., 16(12), 4557-4570.

Burgess, S. S. O., Adams, M. A., Turner, N. C., Beverly, C. R., Ong, C. K., Khan, A. A. H., Bleby, T. M., 2001. An Improved Heat Pulse Method to Measure Low and Reverse Rates of Sap Flow in Woody Plants. Tree Physiol., 21(9), 589-598.

Chen, C., Eamus, D., Cleverly, J., Boulain, N., Cook, P., Zhang, L., Cheng, L., Yu, Q., 2014. Modelling Vegetation Water-use and Groundwater Recharge as Affected by Climate Variability in an Arid-Zone Acacia Savanna Woodland. J. Hydrol., 519(PA), 1084-1096.

Dawes, W., Ali, R., Varma, S., Emelyanova, I., Hodgson, G., McFarlane, D., 2012. Modelling the Effects of Climate and Land Cover Change on Groundwater Recharge in South-West Western Australia. Hydrol. Earth Syst. Sci., 16(8), 2709-2722.

Del Campo, A. D., Fernandes, T. J. G., Molina, A. J., 2014. Hydrology-Oriented (Adaptive) Silviculture in a Semiarid Pine Plantation: How Much can be Modified the Water Cycle through Forest Management? Eur. J. Forest Res., 133(5), 879-894.

Doherty, J., 2009. PEST: Model-Independent Parameter Estimation. Watermark Numerical Guan, H., Simunek, J., Newman, B. D., Wilson, J. L., 2010. Modelling Investigation of Water Partitioning at a Semiarid Ponderosa Pine Hillslope. Hydrol. Process., 24(9), 1095-1105.

Hashemi, H., Berndtsson, R., Kompani-Zare, M., Persson, M., 2013. Natural Vs. Artificial Groundwater Recharge, Quantification through Inverse Modeling. Hydrol. Earth Syst. Sci., 17(2), 637-650.

Hernandez-Santana, V., Asbjornsen, H., Sauer, T., Isenhart, T., Schilling, K., Schultz, R., 2011. Enhanced Transpiration by Riparian Buffer Trees in Response to Advection in a Humid Temperate Agricultural Landscape. For. Ecol. Manage., 261(8), 1415-1427.

Jhorar, R. K., Van Dam, J. C., Bastiaanssen, W. G. M., Feddes, R. A., 2004. Calibration of Effective Soil Hydraulic Parameters of Heterogeneous Soil Profiles. J. Hydrol., 285(14), 233-247.

Legates, D. R., and McCabe Jr., G. J., 1999. Evaluating the use of 'Goodness-of-Fit' Measures in Hydrologic and Hydroclimatic Model Validation. Water Resour. Res., $35(1), 233-241$.

Molina, A. J., and del Campo, A. D., 2012. The Effects of Experimental Thinning on Throughfall and Stemflow: A Contribution Towards Hydrology-Oriented Silviculture in Aleppo Pine Plantations. For. Ecol. Manage., 269, 206-213.

Mualem, Y., 1976. New Model for Predicting the Hydraulic Conductivity of Unsaturated Porous Media. Water Resour. Res., 12(3), 513-522.

Nash, J. E., and Sutcliffe, J. V., 1970. River Flow Forecasting through Conceptual Models Part I - A Discussion of Principles. J. Hydrol., 10(3), 282-290.

Sprintsin, M., Cohen, S., Maseyk, K., Rotenberg, E., Grünzweig, J., Karnieli, A., Berliner, P., Yakir, D., 2011. Long Term and Seasonal Courses of Leaf Area Index in a Semi-Arid Forest Plantation. Agric. for. Meteorol., 151(5), 565-574.

Simunek, J., Sejna, M., Saito, H., Sakai, M., van Genuchten, M.Th., 2013. The HYDRUS1D Software Package for Simulating the Movement of Water, Heat, and Multiple 
Solutes in Variably Saturated Media. Version 4.16. HYDRUS Software Series 3. Dep. Environmental Sciences, Univ. Calif. Riverside, Riverside, CA.

Ungar, E. D., Rotenberg, E., Raz-Yaseef, N., Cohen, S., Yakir, D., Schiller, G., 2013. Transpiration and Annual Water Balance of Aleppo Pine in a Semiarid Region: Implications for Forest Management. For. Ecol. Manage., 298, 39-51.

Van Dijk, A. I. J. M., Hairsine, P. B., Arancibia, J. P., Dowling, T. I., 2007. Reforestation, Water Availability and Stream Salinity: A Multi-Scale Analysis in the Murray-Darling Basin, Australia. For. Ecol. Manage., 251(1-2), 94-109.

Van Genuchten, M. T., 1980. Closed-Form Equation for Predicting the Hydraulic Conductivity of Unsaturated Soils. Soil Sci. Soc. Am. J., 44(5), 892-898.

Wattenbach, M., Zebisch, M., Hattermann, F., Gottschalk, P., Goemann, H., Kreins, P., Badeck, F., Lasch, P., Suckow, F., Wechsung, F., 2007. Hydrological Impact Assessment of Afforestation and Change in Tree-Species Composition - A Regional Case Study for the Federal State of Brandenburg, Germany). J. Hydrol., 346(1-2), 1-17.

Williams, D. G., Cable, W., Hultine, K., Hoedjes, J. C. B., Yepez, E. A., Simonneaux, V., ErRaki, S., Boulet, G., De Bruin, H. A. R., Chehbouni, A., Hartogensis, O. K., Timouk, F., 2004. Evapotranspiration Components Determined by Stable Isotope, Sap Flow and Eddy Covariance Techniques. Agric. for. Meteorol.,125(3-4), 241-258.

Willmott, C. J., 1981. On the Validation of Models. Phys. Geogr., 2(2), 184-194.

Willmott C.J., 1984. On the evaluation of model performance in physical geography, in: Spatial Statistics and Models, edited by: Gaile, G. L. and Willmot, C. J., D. Reidel, Dordrecht, 443-460. 